\title{
miR-205 is frequently downregulated in prostate cancer and acts as a tumor suppressor by inhibiting tumor growth
}

\author{
Ning Wang*, Qi Li*, Ning-Han Feng, Gong Cheng, Zhao-Long Guan, Yang Wang, Chao Qin, Chang-Jun Yin \\ and Li-Xin Hua
}

The purpose of this study was to elucidate the molecular mechanisms of microRNA-205 (miR-205) as a tumor suppressor in prostate cancer (PCa). In the present study, microRNA microarray analysis suggested that the expression of miR-205 was significantly decreased in advanced PCa compared with early PCa. Real-time PCR analysis also indicated that miR-205 expression was significantly decreased in PCa tissues compared with non-cancerous tissues. Moreover, the expression of miR-205 has been demonstrated to be associated with the clinicopathological stage and total/free prostate-specific antigen (PSA) level of PCa. Functional analyses showed that both the overexpression of miR-205 and the knockdown of $c$-SRC in PCa cell lines could inhibit cell growth, colony formation, migration, invasion and the cell cycle as well as induce cell apoptosis in vitro. Furthermore, over-expressing miR-205 reduced tumorigenicity in vivo. Through a luciferase activity assay and Western blotting, $c-S R C$ was identified as a target of miR-205 in cells. The overexpression of miR-205 suppressed c-SRC and its downstream signaling molecules, including FAK, p-FAK, ERK1/2 and p-ERK1/2, and attenuated cell proliferation, invasion and tumor growth.

Asian Journal of Andrology (2013) 15, 735-741; doi:10.1038/aja.2013.80; published online 26 August 2013

Keywords: $c-S R C$; miR-205; prostate cancer (PCa); tumor growth

\section{INTRODUCTION}

Prostate cancer (PCa), which largely affects males, is the second leading cause of cancer-related deaths in the United States, with an estimated 240000 new cases and 33000 deaths in 2011, mainly due to its high potential for tumor progression. ${ }^{1}$ In China, the current PCa incidence rate, although still not very high, has increased dramatically over the past two decades. Moreover, most new cases also suffer symptomatic and metastatic disease because prostate-specific antigen (PSA) and digital rectal examinations are not routinely used to screen PCa in China. ${ }^{2}$ Localized PCa patients have a survival rate of more than 5 years, ${ }^{3,4}$ but this survival rate drops rapidly when tumor progression is detected. ${ }^{5,6}$ Therefore, many attempts have been made to understand the molecular mechanisms of the development and progression of advanced PCa in China so that novel therapeutic strategies may be devised. In pursuit of this objective, novel molecules, one of which is microRNA (miRNA), are being detected and investigated.

miRNAs are small, non-coding RNAs that regulate gene expression by blocking gene translation or decreasing the stability of mRNAs. ${ }^{7,8}$ As a new class of regulatory molecule, miRNAs play critical roles in a variety of biological processes, including cell proliferation, metastasis, development, differentiation, apoptosis, metabolism and immunity. ${ }^{9,10}$ microRNA205 (miR-205) has previously been cited as targeting HER3, SHIP2, VEGF-A, MED1, SIP1 and ZEB1 for repression. ${ }^{11-15}$ In addition, miR-205 has been shown to activate IL24 and IL32 by targeting sites on their promoters. ${ }^{16}$ In PCa, miR-205 exerts a tumorsuppressive effect by counteracting the epithelial-to-mesenchymal transition and reducing cell migration/invasion, in part through the down-regulation of protein kinase $\mathrm{C}$ epsilon. ${ }^{17}$

In this study, we analysed miR-205 expression in advanced and early PCa tissues among Chinese patients using a quantitative real-time PCR assay. By investigating the role of miR-205 in proliferation, cell-cycle progression, apoptosis, migration, invasion and colony formation in DU145 and PC3 cells, we found that forced expression of miR-205 in DU145 cells could inhibit tumor growth in nude mice. Subsequent experiments confirmed that $c-S R C$ was a target gene of miR-205 and was down-regulated by miR-205. Furthermore, that the siRNA-mediated knockdown of $c$-SRC partially phenocopied miR205 restoration suggests that the tumor suppressive role of miR-205 might be mediated through $c-S R C$ regulation. Understanding the functional role of miR-205 could help us to identify new targets and biomarkers that can positively impact patients with PCa.

\section{MATERIALS AND METHODS}

Cell culture and transfection

The human PCa cell lines DU145 and PC3 were purchased from the Shanghai Cell Bank, Chinese Academy of Sciences, and maintained in DMEM supplemented with $10 \%$ foetal bovine serum, 100 units per ml penicillin, and $100 \mu \mathrm{g} \mathrm{ml}^{-1}$ streptomycin in an atmosphere of $5 \% \mathrm{CO}_{2}$ at $37{ }^{\circ} \mathrm{C}$. Has-miRNA-205 mimics, negative controls and siRNAs were chemically designed and synthesized by GenePharma Company (Shanghai, China). Has-miRNA-205 mimics were sense: 5'-UCCUUCAUUCCACCGGAGUCUG-3' and 
anti-sense: 5'-GACUCCGGUGGAAUGAAGAAUU-3'. Negative controls were sense: $5^{\prime}$-UUCUCCGAACGUGUCACGUTT-3' and antisense: $5^{\prime}$-ACGUGACACGUUCGGAGAATT- $3^{\prime}$. siRNA-SRC (S1) were sense: $5^{\prime}$-CUCGGCUCAUUGAAGACAATT- $3^{\prime}$ and anti-sense: $5^{\prime}$-UUGUCUUCAAUGAGCCGAGTT- $3^{\prime}$. Cells of $50 \%-60 \%$ confluence were transfected with oligonucleotides using Lipofectamine 2000 reagent (Invitrogen, Carlsbad, CA, USA).

\section{miRNA microarray}

Fluorescence targets were taken from $2.5 \mu \mathrm{g}$ total RNA samples using the miRNA ULS Labeling Kit (Kreatech Diagnostics, Amsterdam, The Netherlands). The miRNA microarray analysis was carried out by a commercial company (Phalanx Biotech, Hsinchu, Taiwan, China) using human miRNA OneArray v3. The Cy5 fluorescent intensities of each spot were analysed by GenePix 4.1 software (Molecular Devices, Silicon Valley, CA, USA).

\section{Quantitative real-time PCR}

Total RNA was extracted and assayed for mature miRNAs. TaqMan MicroRNA Assays (Applied Biosystems, Carlsbad, CA, USA) were introduced in accordance with the manufacturer's instructions. All real time reactions were run in a StepOnePlus Real Time PCR System (Applied Biosystems). Relative expressions were calculated by the $2^{-\Delta C_{t}}$ method.

\section{Cell proliferation assay}

DU145 and PC3 cells with miR-205 mimics, $c$-SRC-siRNA or negative control (NC) that had been transfected for $48 \mathrm{~h}$ were seeded into 96well plates (2000 cells per well) and cultured for 24, 48, 72 and $96 \mathrm{~h}$. Each well received $20 \mu \mathrm{l}$ of CCK- 8 solution in addition to the $200 \mu \mathrm{l}$ of culture media, and then the plate was incubated for $1.5 \mathrm{~h}$ at $37{ }^{\circ} \mathrm{C}$. Absorbance at $450 \mathrm{~nm}$ was measured by a $V_{\max }$ microplate spectrophotometer (Molecular Devices).

\section{Colony formation assay}

Regarding the colony formation assay, cells were seeded into six-well plates at a low density ( 200 cells per plate) and cultured for 14 days. Then, the cells were fixed with $95 \%$ methanol and pigmented with $0.1 \%$ crystal violet. The number of colonies was counted.

\section{Migration and invasion assays}

Cell migration and invasion were assayed in 24-well plates using a chamber with a $6.5-\mathrm{mm}$ diameter and an $8-\mu \mathrm{m}$ pore size (BD Biosciences, Silicon Valley, CA, USA). Forty-eight hours post-transfection, DU145 and PC3 cells were added to the upper chamber, which were coated with $1 \mathrm{mg} \mathrm{ml}^{-1}$ matrigel for the invasion assays, and $0.7 \mathrm{ml}$ of $20 \%$ FBSDMEM was added to the lower chamber. After $48 \mathrm{~h}$ of incubation, the cells in the upper chamber were removed, while the migrated or invaded cells were fixed with $95 \%$ methanol, stained with $0.1 \%$ crystal violet, and photographed in five independent fields for each well.

\section{Flow cytometry analysis of cell cycle and apoptosis}

For the cell cycle analysis, $48 \mathrm{~h}$ after transfection, DU 145 and PC 3 cells were fixed with $75 \%$ cold ethanol at $-20{ }^{\circ} \mathrm{C}$ for $24 \mathrm{~h}$. Propidium iodide and RNase A (Sigma, San Francisco, CA, USA) were added to the cells. The samples were analysed $30 \mathrm{~min}$ after staining using the flow cytometry-BD FACSCalibur (BD Biosciences) and CellQuest software. For the apoptosis assays, the apoptosis rates were determined by Annexin V-FITC and PI (KeyGen, Nanjing, China) staining flow cytometry.

\section{Luciferase reporter assay}

Human $c$-SRC $3^{\prime}$-UTR was amplified and cloned into the XbaI site of the pGL3-control vector (Invitrogen), downstream of the luciferase gene, to generate the plasmids pGL3-WT-c-SRC-3'-UTR. pGL3-Mut$c$-SRC-3'-UTR was generated from pGL3-WT-c-SRC-3'-UTR by changing two base pairs of the miR-205 seed-sequence. For the luciferase reporter assays, the cells were cotransfected with the wild-type or mutant reporter plasmid and the miR-205 or negative control. Luciferase activities were analysed by dual luciferase assays (Promega, Madison, WI, USA) $48 \mathrm{~h}$ after cotransfection and normalized against the activity of the Renilla luciferase gene.

\section{Tumorigenicity assays in nude mice}

BALB/C nu/nu male mice (4-6 weeks) were purchased from Shanghai SLAC Laboratory Animals. All experimental procedures involving animals followed the National Institute of Health Guide for the Care and Use of Laboratory Animals and were approved by the ethical committee of the First Affiliated Hospital of Nanjing Medical University. Aliquots of DU145 cells $\left(1 \times 10^{6}\right.$ cells in $\left.50 \mu \mathrm{l}\right)$ transfected with miR-205 or NC were mixed with $50 \mu$ l Matrigel (BD Biosciences) and injected subcutaneously into each side of the flanks of five male nude mice. These mice were then treated with 200 pmol miR-205 or NC mimics in $10 \mu \mathrm{l}$ Lipofectamine 2000 through a local injection of the xenograft tumor every seven days. The tumor size was measured every 4 days with callipers. Twenty-eight days later, the tumours were removed, stained with antibody against Ki-67 (Abcam, Cambridge, $\mathrm{UK})$, and microscopically visualized.

\section{Western blot analysis}

Proteins were separated by $10 \%$ SDS polyacrylamide gels and electrophoretically transferred to polyvinylidene difluoride membranes (Millipore, Billerica, MA, USA). The membranes were blocked overnight with $5 \%$ non-fat dried milk and incubated for $2 \mathrm{~h}$ with antibodies to c-SRC, ERK1/2, p-ERK1/2 (Cell Signaling Technology, Beverly, MA, USA), p-c-SRC, FAK, p-FAK, c-MYC, CYCLIND1 (Bioworld, Nanjing, China) and GAPDH (Bioworld). The signals were detected by enhanced chemiluminescence (Millipore) after incubation with goat anti-rabbit secondary antibody (Bioworld).

\section{Statistical analysis}

All values in the study were reported as the means \pm s.d. All of the experiments above were repeated three times. The relationship between down-regulated miR-205 expression and total PSA or free PSA in PCa was analysed using the Spearman rank correlation test. All other data were compared by two-sided $t$ - tests. Statistical analysis was performed by SPSS v. 17.0 computer software. $P<0.05$ were considered statistically significant.

\section{RESULTS}

MiR-205 is significantly downregulated in PCa tissues

To confirm the expression of miRNAs in the PCa tissue samples, we performed microarray experiments using a mixed RNA extract from three advanced PCa tissues (clinical stage $\geqslant \mathrm{T} 3$ ) and another mixed RNA extract from three early PCa tissues (clinical stage $\leqslant \mathrm{T} 2$ ). We found that miR-205 was more significantly down-regulated in advanced PCa (Figure 1a). In addition, to validate the results of the microarrays, we examined the expression of miR-205 in 18 noncancerous tissues, 15 T2 stage PCa tissues and 13 T3-T4 stage tissues by qRT-PCR. As expected, the results showed that miR-205 expression was significantly weakened in PCa tissues compared with non-cancerous 
a

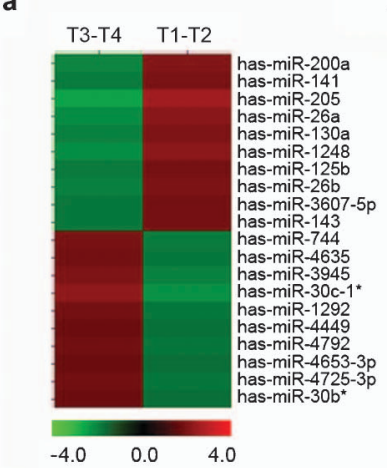

c

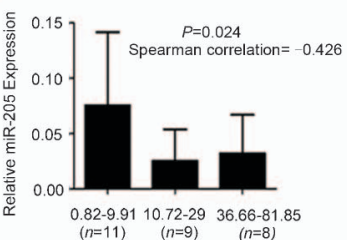

b
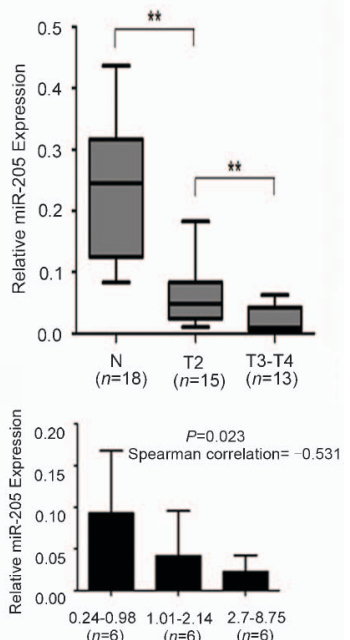

Figure 1 Validation of the selected miR-205 that was predicted to be downregulated in advanced prostate cancer. (a) A heat map comparing the average fold-changes in microRNAs with markedly lower (green) or higher (red) expression in advanced PCa (clinical stage T3-T4) in comparison with early PCa (clinical stage T1-T2), as determined by microarray analysis. (b) The expression levels of miR-205 were analysed in 13 advanced prostate cancer tissues, 15 early prostate cancer tissues and 18 non-cancerous tissues by qRT-PCR. $* * P<0.01$. (c) Tumor samples were divided into three groups with approximately equal sample sizes based on the levels of total or free PSA. The level of total or free PSA in patients with primary tumours is presented on the $x$ axis. The $y$ axis indicates the total expression of miR-205. There was a statistically significant Spearman correlation that characterized an inverse relationship between miR-205 expression and total or free PSA (Spearman correlation $=-0.426, P=0.024$; Spearman correlation $=-0.531, P=0.023)$. miR-205, microRNA-205; PCa, prostate cancer; PSA, prostate-specific antigen.

tissues $(P<0.01, t$-test; Figure $\mathbf{1 b})$. The results also indicated that miR-205 expression possibly correlated with the clinicopathological stage of PCa (Figure 1b). Moreover, the results showed significant inverse correlations between the expression of miR-205 and total PSA levels (Spearman correlation $=-0.426, P=0.024$; Figure 1c), as well as a significant inverse correlation between the expression of miR-205 and free PSA levels (Spearman correlation $=-0.531, P=0.023$; Figure 1c). The clinical characteristics of the study's PCa population are listed in Table 1

Table 1 Clinical characteristics of the study's prostate cancer population $(n=28)$

\begin{tabular}{lc}
\hline & Values \\
\hline Age (year) & \\
Mean (range) & $70(56-78)$ \\
Clinical stage & 15 \\
T2 & 10 \\
T3 & 3 \\
T4 & \\
Total PSA at diagnosis & $24.91(0.82-81.85)$ \\
Median (range) & \\
Free PSA at diagnosis & $2.16(0.24-8.75)$ \\
Median (range), $n=18$ & \\
\hline
\end{tabular}

Abbreviations: PCa, prostate cancer; PSA, prostate-specific antigen.

\section{MiR-205 suppresses tumorigenicity in vitro}

The significant reduction of miR-205 expression in PCa tissues prompted us to explore the possible biological function of miR-205 in tumorigenesis. The results demonstrated that the proliferative capacity of PC 3 and DU145 cells, when transfected with miR-205 mimics, significantly declined in comparison with cells transfected with NC $(P<0.05, t$-test; Figure 2a). As shown in figure 2b, compared with NC transfectants, miR-205-restored PC3 and DU145 cells also had lower colony formation abilities $(P<0.01, t$-test $)$. To further examine whether the decrease in the proliferation of PC3 and DU145 cells reflected cell-cycle arrest and the induction of apoptosis, cell cycle and apoptosis progression were analyzed by flow cytometry. The results revealed that miR-205-restored PC3 and DU145 cells had significant increases in the $G_{0} / G_{1}$ phase of their cell cycles compared with NC transfectants $(P<0.01, t$-test, Figure $2 \mathrm{c})$. On the other hand, the percentage of total apoptotic cells significantly increased in response to miR-205 transfection, compared with $\mathrm{NC}$, with a corresponding decrease close to $10 \%$ in the viable cell population $(P<0.01, t$-test; Figure 2d).

\section{MiR-205 inhibited PCa cell migration and invasion}

Transwell migration and invasion assays were used to investigate whether miR-205-restored PC3 and DU145 cells correlated with tumor metastasis. As shown in figure $2 \mathbf{e}$, when the expression of miR-205 was upregulated by mimics-miR-205 in the PC3 and DU145 cell lines, the cells demonstrated low-metastasis potentiality compared with cells treated with $\mathrm{NC}(P<0.05, t$-test $)$. All of these results suggested a potential tumor suppressor role for miR-205 in $\mathrm{PCa}$ in vitro.

\section{Knockdown of $c$-SRC mimicked miR-205 inhibition}

To determine whether the downregulation of $c-S R C$ is involved in miR-205-induced functional assays, we analysed the knockdown effect of $c$-SRC in PCa cells. We initially tested three siRNAs to knockdown the $c-S R C$ gene and then confirmed the results at varying protein levels (Figure 3a). Then, we chose one siRNA (S-1) for additional experiments. As expected, compared with NC, $c$-SRC-knockdown DU145 and PC3 cells showed decreases in their proliferation, migration and invasion and increases in their G1-phase population and cell apoptosis (Figure 3b-3e), similar to the phenotype observed upon miR-205 restoration.

\section{$c-S R C$ was targeted by miR-205}

To determine whether $c$-SRC was a bona fide target of miR-205, the human $c$-SRC 3'-UTR fragment containing wild-type or mutant miR205-binding sequences was subcloned to the downstream Renilla luciferase reporter gene. The binding site was conserved in the region complementary to the seed sequence (Figure 3a). When miR-205 mimics were cotransfected with the reporter plasmids, the relative luciferase activity of the reporter containing wild-type $c$-SRC $3^{\prime}$ UTR was obviously suppressed, while the luciferase activity of the reporter containing mutant $c$-SRC $3^{\prime}$-UTR was unaltered (Figure 4a). These results indicated that $c-S R C$ was a direct target of miR-205, with specific binding sites at the seed sequences. To further ascertain the relationship between miR-205 and c-SRC expression, Western blots were performed to examine the effect of the overexpression of miR-205 on the c-SRC protein levels in PCa cells. As expected, the overexpression of miR-205 suppressed c-SRC and p-cSRC expression at the protein level in DU145 and PC3 cells (Figure 4b). 


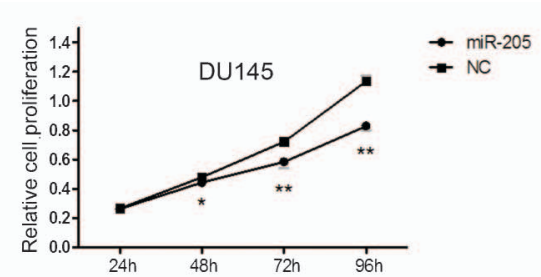

b

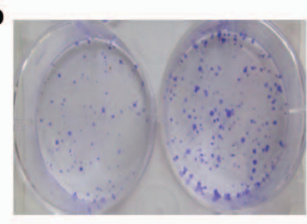

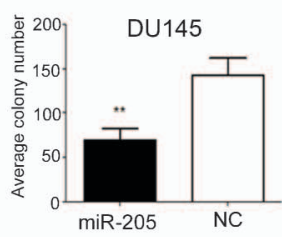

DU145
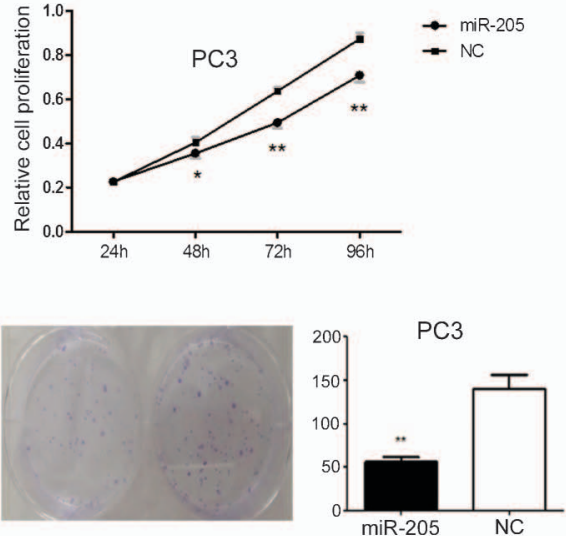

PC3
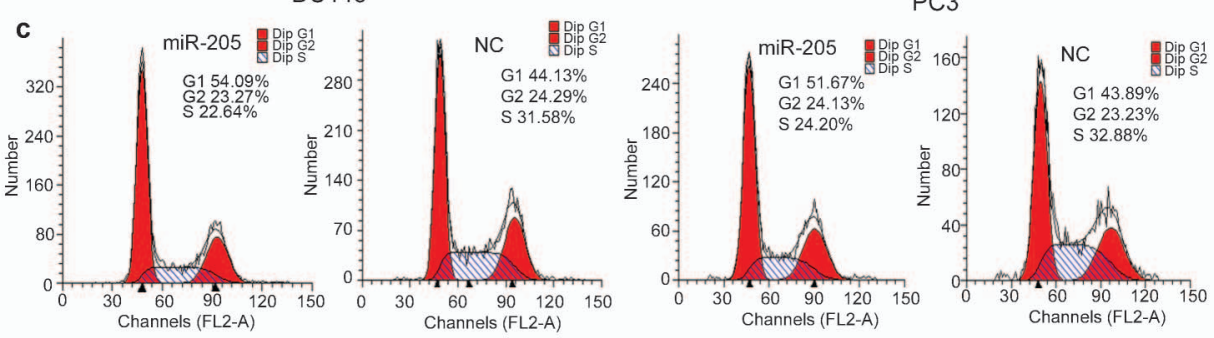

d

DU145
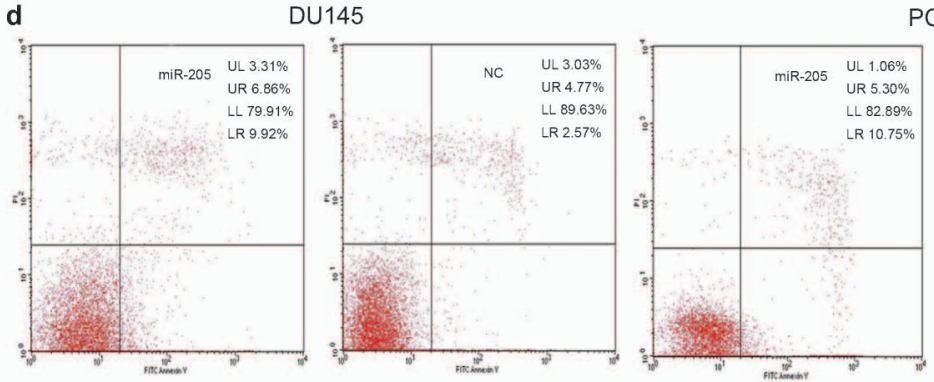

PC3
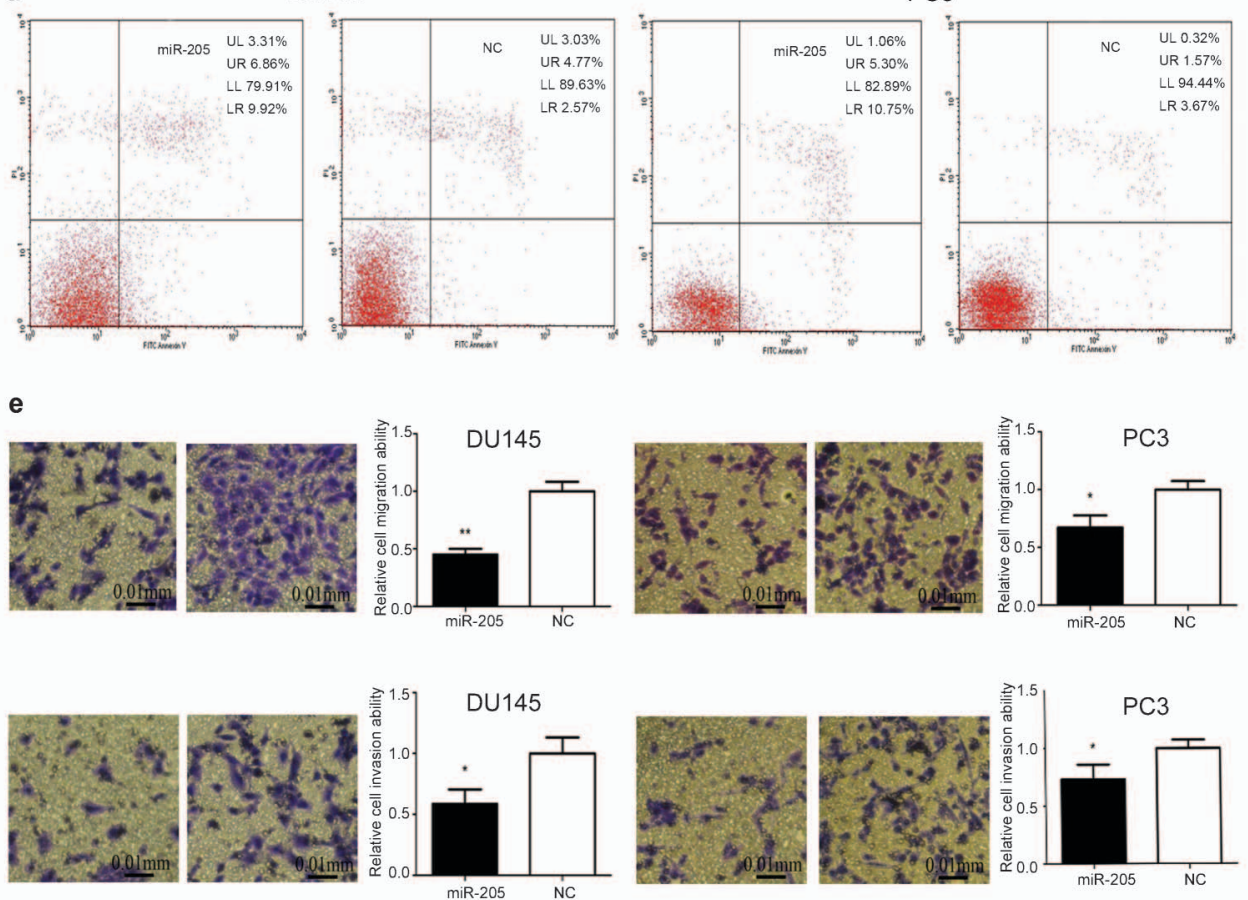

Figure 2 Effect of miR-205 on cell proliferation, colony formation, cell cycle, apoptosis, migration and invasion of DU145 and PC3 cells in vitro. (a) miR-205 overexpression significantly inhibited the proliferation of DU145 and PC3 cells. (b) Colony formation of DU145 and PC3 cells after miR-205 transfection was significantly reduced compared with NC. (c) The forced expression of miR-205 led to a significant increase in the $G_{0} / G_{1}$ phase of the cell cycle compared with NC. (d) The forced expression of miR-205 increased the sensitivity of DU145 and PC3 cells to apoptosis. (e) The forced expression of miR-205 suppressed the migratory capacity and invasive capacity of DU145 and PC3 cells. ${ }^{*} P<0.05,{ }^{* *} P<0.01$. Scale bar $=0.01 \mathrm{~mm}$. LL, normal cells; LR, late apoptotic cells; miR-205, microrna-205; NC, negative control; UL, dead cells; UR, early apoptotic cells.

\section{miR-205 inhibits the FAK/ERK1/2 pathway}

To investigate the role of miR-205 in the regulation of cellular signaling, we performed Western blotting for the downstream genes of miR-205 in the DU145 and PC3 cells that were either
miR-205- or NC-transfected. We found that transient transfection with miR-205 down-regulated FAK, p-FAK, ERK1/2 and p-ERK $1 / 2$ in the form of proteins (Figure $4 \mathbf{b}$ ). These data indicated that miR-205 targets $c$-SRC, resulting in suppression of the 
a

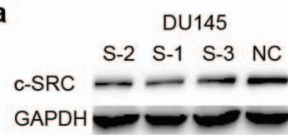

Has-miR-205 3'-G-UCUGAGGCCACCUUACUUCCU-5'

c-SRC wt 5'-CAGGCCCUGCCAGUGGGGAAGGA-3'

c-SRC mut 5'-CAGGCCCUGCCAGUGGGGAACCA-3' b

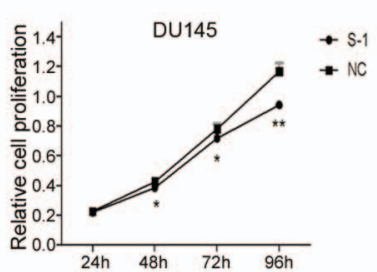

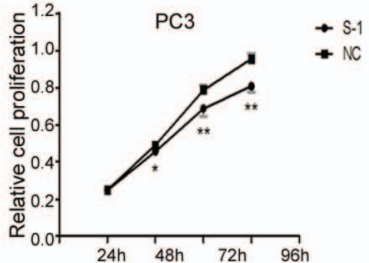

$+\mathrm{NC}$

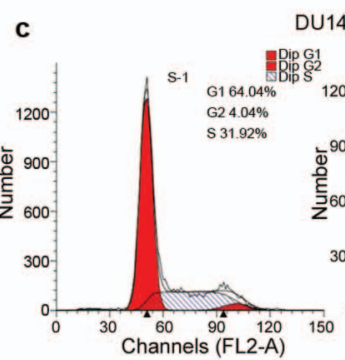

DU145

d

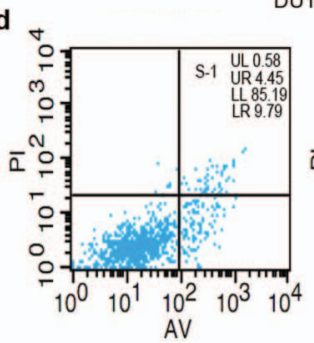

DU145
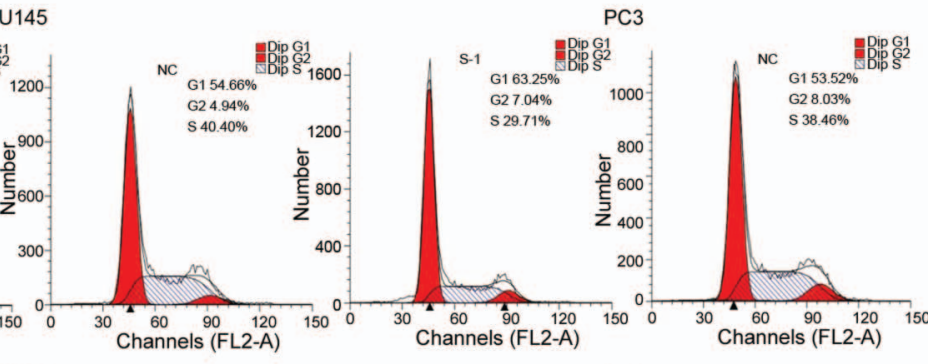

PC3
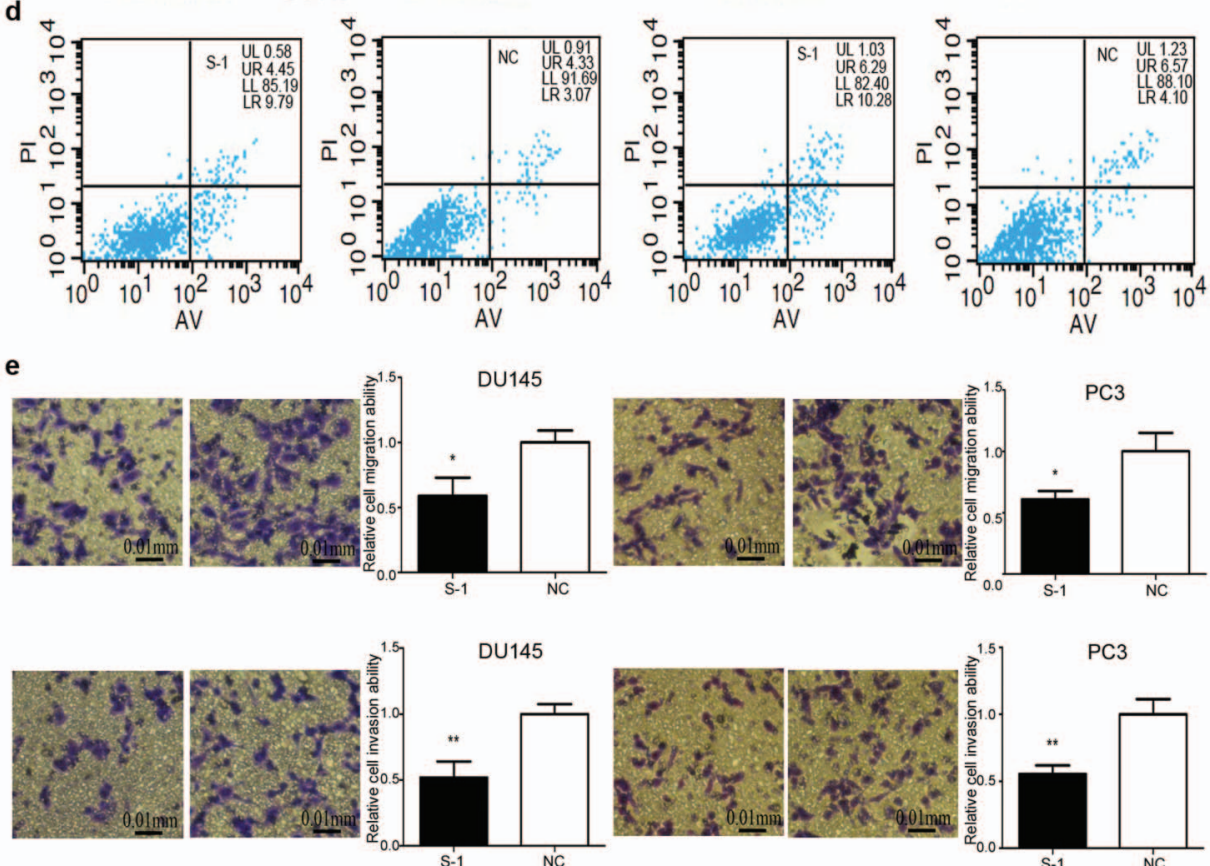

Figure 3 Knockdown of $c-S R C$ mimicked miR-205 inhibition in functional tests. (a) c-SRC protein levels were assessed by Western blot in DU145 cells transfected with $c$-SRC-siRNA (S-1, S-2 and S-3) and NC. The c-SRC 3'-UTR was cloned into the pGL3-control vector (WT), and the mutant construct was obtained by sitedirected mutagenesis, which changed two base pairs of the miR-205 seed-sequence (Mut) and inserted them into the vector. (b) c-SRC-knockdown DU145 and PC3 cells showed decreased proliferation compared with NC. (c) Knockdown of $c$-SRCled to a significant increase in the $\mathrm{G}_{0} / \mathrm{G}_{1}$ phase of the cell cycle compared with NC. (d) Knockdown of $c$-SRC increased the sensitivity of DU145 and PC3 cells to apoptosis. (e) Knockdown of $c$-SRC suppressed the migratory and invasive capacities of DU145 and PC3 cells. $* P<0.05, * * P<0.01$. Scale bar $=0.01$ mm. miR-205, microrna-205; NC, negative control.

FAK/ERK1/2 pathway and the genes involved in proliferation. We also found that transient transfection with miR-205 decreased the expression of the cycle-related proteins, c-MYC and CYCLIND1 (Figure 4b).

\section{MiR-205 suppresses tumor growth in vivo}

As expected, the miR-205-restored DU145 cells exhibited a delay in palpable tumor onset and a marked degeneration in tumor growth when compared with NC $(P<0.05, t$-test; Figure $4 c)$. In addition, miR-205 reduced Ki-67 staining in tumor xenografts $(P<0.01, t$-test;
Figure 4d), suggesting that miR-205 reduced tumorigenicity in a nude mouse model.

\section{DISCUSSION}

miRNAs are a class of small RNAs that regulate various physiological and pathological mechanisms. ${ }^{18}$ Although upregulated in ovarian, bladder and breast carcinomas, ${ }^{19-21}$ miR-205 has been reported to be downregulated in oesophageal cancers. ${ }^{22}$ Thus, miR-205 might act as a doubled-edged sword by targeting opposite functional genes, as one miRNA can target a dozen mRNAs, impacting many molecules 


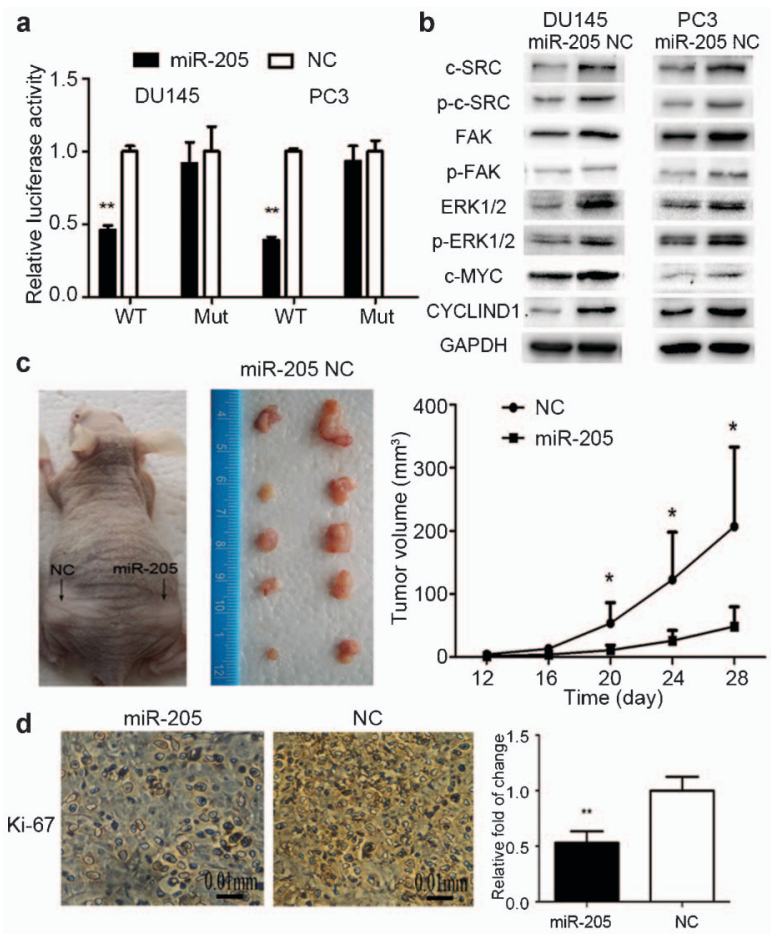

Figure $4 c-S R C$ is a potential target of miR-205. (a) The relative luciferase activities of $c-S R C$ wild type (WT) and mutant (Mut) $3^{\prime}$-UTR regions were obtained by the cotransfection of miR-205 or NC and the pRL-TK plasmid. The ratio of the firefly/renilla activities was calculated in the DU145 and PC3 cells and normalized to that of the controls. The results were presented as the means \pm s.d. of each experiment, in triplicate. ${ }^{*} P<0.01$. (b) Overexpression of miR-205 suppressed c-SRC and $p-c-S R C$, as well as the expression of its downstream signaling molecules, FAK, p-FAK, ERK1/2 and p-ERK1/2, at the protein level in DU145 and PC3 cells. Overexpression of miR-205 also suppressed the cyclerelated proteins C-MYC and CYCLIND1. (c) The tumor volume of the miR-205 group was decreased compared with the NC group. (d) miR-205 reduced Ki-67 immunostaining in tumours. $* P<0.05, * * P<0.01$. Scale bars $=0.01 \mathrm{~mm}$. miR205, microRNA-205; NC, negative control.

in different signal pathways. Here, we used qRT-PCR to profile the expression of miR-205 in PCa and non-cancerous tissues. Consistent with previous studies, ${ }^{16,17}$ we demonstrated that the expression of miR-205 in PCa tissues was down-regulated when compared with non-cancerous tissues (Figure 1b). Furthermore, miR-205 was shown to be downregulated in advanced-stage PCa in our studies (Figure 1a and $\mathbf{1 b}$ ) and to be down-regulated in metastatic $\mathrm{PCa} .{ }^{23}$ Chung et al. ${ }^{24}$ reported that the aberrant expression of miR-205 took place in advanced stages in endometrial cancer. In addition, previous studies have shown that the level of miR-205 was inversely correlated with PSA and tumor stage. ${ }^{25,26}$ In our study, the results showed that miR205 expression was also related to the stage of the disease (Figure 1b). We further found that the expression level of miR-205 was inversely correlated with the total/free PSA levels (Figure 1c). These results indicated that, as a tumor suppressor gene, miR-205 may be used not only for an early diagnosis of PCa but also as a marker of tumor progression.

Deregulated cell proliferation is a key mechanism in neoplastic progression. ${ }^{27}$ Our cell proliferation assay showed that miR-205 overexpression in PCa cells markedly attenuated cell proliferation (Figure 2a). In the present study, we found that PCa cells transfected with miR-205 mimics had obvious cell cycle arrest at the $G_{0} / G_{1}$ phase, coupled with a decrease in cellular apoptosis, as shown by the flow cytometry analysis (Figure $\mathbf{2 c}$ and $\mathbf{2 d}$ ). However, whether miR-205 could affect the proliferation of PCa cells remains controversial. Gandellini et al. ${ }^{17}$ reported that the aberrant expression of miR-205 did not induce appreciable perturbations of cell proliferation or cell cycle progression. However, a previous report described that miR-205 impaired tumor cell growth by the induction of apoptosis and cell cycle arrest. We used nude mouse xenograft models to confirm that miR-205 could suppress the tumorigenicity of PCa cells. Our results clearly demonstrated that miR-205 overexpression in DU145 cells delayed xenograft tumor growth, highlighting the relevance of miR205 as a putative therapeutic agent for the treatment of this disease (Figure 4c). Furthermore, to confirm the indications that miR-205 may reduce tumor xenograft growth by decreasing proliferation, we evaluated proliferation in tumor xenograft tissue sections by Ki-67 immunohistochemistry. As expected, miR-205 overexpression markedly decreased the expression of Ki-67, which was consistent with the marked reduction in tumor growth (Figure 4d). To our knowledge, this is the first demonstration that miR-205 overexpression decreases proliferation in human PCa cells xenografted into mice.

The loss of miR-205 is associated with enhanced metastatic potential in both model tumor systems and in human cancers, particularly of the breast. ${ }^{13,15}$ miR-205 may halt PCa progression through the downregulation of protein kinase $\mathrm{C}$ epsilon. ${ }^{17}$ Regarding the human PCa cell lines DU145 and PC3, which have a high metastatic ability and low expression of miR-205, ${ }^{17}$ the upregulation of the expression of miR-205 permitted these PCa cell lines to gain low metastatic potentiality (Figure 2e). Further, we concluded that the siRNA knockdown of $c$-SRC inhibited cell proliferation, impairing the cells' invasion and migration abilities and inducing $\mathrm{G}_{0} / \mathrm{G}_{1}$ arrest and late apoptosis in PCa cell lines (Figure $\mathbf{3 b}-\mathbf{3 e}$ ). All of these results suggested that miR205 may function as a tumor suppressor, partially through the downregulation of c-SRC expression.

The SRC family kinases (SFKs) are non-receptor tyrosine kinases and prototypical modular signaling proteins. ${ }^{28-30}$ The SFKs play key roles in cell proliferation, invasion, migration, apoptosis, survival, adhesion, morphology and motility. ${ }^{29,31,32}$ Among the SFKs, c-SRC is arguably the best characterized and most often implicated $S F K$ in cancer. Advanced stages of cancer progression have been associated with high $c$-SRC expression. ${ }^{33}$ Increased $c$-SRC activity takes place in bladder carcinoma, ${ }^{34}$ head and neck, ${ }^{35}$ colon $^{36}$ and breast ${ }^{37}$ cancers. Majid et al. ${ }^{38}$ reported that the $S R C$ family members were direct targets of miR-205 in renal cancer. In PCa, we also confirmed that $c-S R C$ was a target of miR-205, with the forced expression of miR-205 suppressing $\mathrm{c}-\mathrm{SRC}$ and p-c-SRC protein expression (Figure $4 \mathrm{~b}$ ). Moreover, the $c$ $S R C 3^{\prime}$-UTR reporter experiment showed a marked decline in luciferase activity after miR-205 over-expression (Figure 4a).

The remarkable influence of miRNA on the regulation of cell function may depend on the essential target genes involved in different signal pathways. In this model, miR-205 targeted $c$-SRC and its downstream signal pathway, which play a major role in the regulation of tumor cell growth. It has been reported that $c-S R C$ is involved in multiple signaling pathways, including RAS/RAF/ERK1/2, PI3K/AKT/ HIF-1 $\alpha$, STAT3/c-MYC/CYCLIND1, B-CATENIN/c-MYC/CYCLIND1, FAK/p130CAS/MMP9 and RAC/NADPH, which induce the growth, survival and migration of various types of cancer cells. ${ }^{39-41}$ The ability to target $c-S R C$ transcripts signifies that miR-205 might be a potential regulator of the FAK/ERK1/2 pathways in PCa cells. Western blot analysis showed that the expression levels of the members of the FAK/ ERK1/2 pathway were reduced through targeting with c-SRC after miR-205 overexpression (Figure $4 \mathbf{b}$ ). Moreover, our results showed 
that miR-205 can inhibit the constitutive activity of p-FAK, p-ERK1/2, c-MYC and CYCLIND1 in PCa cells with suppressed p-c-SRC expression (Figure $4 b$ ).

We concluded that there was markedly low expression of miR-205 in PCa tissues compared with non-cancerous tissues. Moreover, the expression of miR-205 was found to be associated with the clinicopathological stage and level of the total PSA/free PSA in PCa patients. Our results clearly confirmed that miR-205 suppressed the proliferation and metastasis of the PCa cells by targeting $c$-SRC through the $F A K / E R K 1 / 2$ pathway. As no effective approaches for curing advanced PCa exist at present, we propose that gene therapy targeting miR-205/ $c$-SRC could serve as a potential therapeutic strategy for PCa.

\section{AUTHOR CONTRIBUTIONS}

All authors discussed the results and implications and commented on the manuscript at all stages. LXH, CJY and CQ conceived and designed the study. NW, NHF and GC gathered and analyzed the data. NW, QL, ZLG and YW performed the experiment. NW wrote and revised the paper, which was read and approved by all authors.

\section{COMPETING FINANCIAL INTERESTS}

All authors declare that there are no competing financial interests.

\section{ACKNOWLEDGMENTS}

This study was supported by the Program for Development of Innovative Research Team in the First Affiliated Hospital of Nanjing Medical University, Provincial Initiative Program for Excellency Disciplines (No. 81171963), the National Nature Science Foundation of China (No. 81272831/H1619), the Natural Science Foundation of Jiangsu Province (No. BK2010577) and Jiangsu Province's Outstanding Medical Academic Leader program (No. RC201178).

1 Siegel R, Ward E, Brawley O, Jemal A. Cancer statistics, 2011: the impact of eliminating socioeconomic and racial disparities on premature cancer deaths. $C A$ Cancer J Clin 2011; 61: 212-36.

2 Dai B, Ye DW, Kong YY, Shen YJ, Wang BH. Individualized prostate biopsy strategy for Chinese patients with different prostate specific antigen levels. Asian J Andro/ 2008; 10: 325-31.

3 Klotz L. Active surveillance for prostate cancer: for whom?. J Clin Oncol 2005; 23: 8165-9.

4 Walz J, Gallina A, Saad F, Montorsi F, Perrotte P et al. A nomogram predicting 10-year life expectancy in candidates for radical prostatectomy or radiotherapy for prostate cancer. J Clin Oncol 2007; 25: 3576-81.

5 Sabbatini P, Larson S, Kremer A, Zhang Z, Sun M et al. Prognostic significance of extent of disease in bone in patients with androgen-independent prostate cancer. $J$ Clin Oncol 1999; 17: 948-57.

6 Loberg R, Gayed B, Olson K, Pienta K. A paradigm for the treatment of prostate cancer bone metastases based on an understanding of tumor cell-microenvironment interactions. J Cell Biochem 2005; 96: 439-46.

7 Zamore PD, Haley B. Ribo-gnome: the big world of small RNAs. Science 2005; 309 . 1519-24

8 Mattick JS, Makunin IV. Non-coding RNA. Hum Mol Genet 2006; 15: R17-29.

9 Chen CZ, Li L, Lodish HF, Bartel DP. MicroRNAs modulate hematopoietic lineage differentiation. Science 2004; 303: 83-6.

$10 \mathrm{Kim}$ VN. MicroRNA biogenesis: coordinated cropping and dicing. Nat Rev Mol Cell Biol 2005; 6: 376-85

11 Iorio MV, Casalini P, Piovan C, Di Leva G, Merlo A et al. microRNA-205 regulates HER3 in human breast cancer. Cancer Res 2009; 69: 2195-200.
12 Yu J, Ryan DG, Getsios S, Oliveira-Fernandes M, Fatima A et al. MicroRNA-184 antagonizes microRNA-205 to maintain SHIP2 levels in epithelia. Proc Natl Acad Sci USA 2008; 105: 19300-5.

13 Wu H, Zhu S, Mo YY. Suppression of cell growth and invasion by miR-205 in breast cancer. Cell Res 2009; 19: 439-48.

14 Mouillet JF, Chu T, Nelson DM, Mishima T, Sadovsky Y. MiR-205 silences MED1 in hypoxic primary human trophoblasts. FASEB J 2010; 24: 2030-9.

15 Gregory PA, Bert AG, Paterson EL, Barry SC, Tsykin A et al. The miR-200 family and miR-205 regulate epithelial to mesenchymal transition by targeting ZEB1 and SIP1. Nat Cell Biol 2008; 10: 593-601.

16 Majid S, Dar AA, Saini S, Yamamura S, Hirata H et al. MicroRNA-205-directed transcriptional activation of tumor suppressor genes in prostate cancer. Cancer 2010; 116: 5637-49.

17 Gandellini $\mathrm{P}$, Folini M, Longoni N, Pennati M, Binda M et al. miR-205 Exerts tumorsuppressive functions in human prostate through down-regulation of protein kinase Cepsilon. Cancer Res 2009; 69: 2287-95.

18 Mendell JT. MicroRNAs: critical regulators of development, cellular physiology and malignancy. Cell Cycle 2005; 4: 1179-84.

19 Iorio MV, Visone R, Di Leva G, Donati V, Petrocca F et al. MicroRNA signatures in human ovarian cancer. Cancer Res 2007; 67: 8699-707.

20 Gottardo F, Liu CG, Ferracin M, Calin GA, Fassan M et al. Micro-RNA profiling in kidney and bladder cancers. Urol Oncol 2007; 25: 387-92.

21 Iorio MV, Ferracin M, Liu CG, Veronese A, Spizzo R et al. MicroRNA gene expression deregulation in human breast cancer. Cancer Res 2005; 65: 7065-70.

22 Feber A, Xi L, Luketich JD, Pennathur A, Landreneau RJ et al. MicroRNA expression profiles of esophageal cancer. J Thorac Cardiovasc Surg 2008; 135: 255-60.

23 Watahiki A, Wang Y, Morris J, Dennis K, O'Dwyer HM et al. MicroRNAs associated with metastatic prostate cancer. PLOS ONE 2011; 6: e24950.

24 Chung TK, Cheung TH, Huen NY, Wong KW, Lo KW et al. Dysregulated microRNAs and their predicted targets associated with endometrioid endometrial adenocarcinoma in Hong Kong women. Int J Cancer 2009; 124: 1358-65.

25 Hagman Z, Haflidadottir BS, Ceder JA, Larne O, Bjartell A et al. miR-205 negatively regulates the androgen receptor and is associated with adverse outcome of prostate cancer patients. Br J Cancer 2013; 108: 1668-76.

26 Schaefer A, Jung M, Mollenkopf HJ, Wagner I, Stephan C et al. Diagnostic and prognostic implications of microRNA profiling in prostate carcinoma. Int J Cancer 2010; 126: 1166-76.

27 Evan GI, Vousden KH. Proliferation, cell cycle and apoptosis in cancer. Nature 2001 411: 342-8.

28 Summy JM, Gallick GE. Src family kinases in tumor progression and metastasis. Cancer Metastasis Rev 2003; 22: 337-58.

29 Thomas SM, Brugge JS. Cellular functions regulated by Src family kinases. Annu Rev Cell Dev Biol 1997; 13: 513-609.

30 Martin GS. The hunting of the Src. Nat Rev Mol Cell Biol 2001; 2: 467-75.

31 Yeatman TJ. A renaissance for SRC. Nat Rev Cancer 2004; 4: 470-80.

32 Frame MC. Src in cancer: deregulation and consequences for cell behaviour. Biochim Biophys Acta 2002; 1602: 114-30.

33 Ishizawar R, Parsons SJ. c-Src and cooperating partners in human cancer. Cancer Cell 2004; 6: 209-14.

34 Boyer B, Bourgeois Y, Poupon MF. Src kinase contributes to the metastatic spread of carcinoma cells. Oncogene 2002; 21: 2347-56.

35 Mandal M, Myers JN, Lippman SM, Johnson FM, Williams MD et al. Epithelial to mesenchymal transition in head and neck squamous carcinoma: association of Src activation with E-cadherin down-regulation, vimentin expression, and aggressive tumor features. Cancer 2008; 112: 2088-100.

36 Aligayer H, Boyd DD, Heiss MM, Abdalla EK, Curley SA et al. Activation of Src kinase in primary colorectal carcinoma: an indicator of poor clinical prognosis. Cancer 2002, 94: 344-51.

37 Reissig D, Clement J, Sänger J, Berndt A, Kosmehl H et al. Elevated activity and expression of Src-family kinases in human breast carcinoma tissue versus matched non-tumor tissue. J Cancer Res Clin Oncol 2001; 127: 226-30.

38 Majid S, Saini S, Dar AA, Hirata H, Shahryari V et al. MicroRNA-205 inhibits Src-mediated oncogenic pathways in renal cancer. Cancer Res 2011; 71: 2611-21.

39 Chang YM, Bai L, Liu S, Yang JC, Kung HJ et al. Src family kinase oncogenic potential and pathways in prostate cancer as revealed by AZD0530. Oncogene 2008; 27: 6365-75.

40 Morin PJ. beta-catenin signaling and cancer. Bioessays 1999; 21: 1021-30.

41 Prathapam T, Tegen S, Oskarsson T, Trumpp A, Martin GS. Activated Src abrogates the Myc requirement for the $\mathrm{G}_{0} / \mathrm{G}_{1}$ transition but not for the $\mathrm{G}_{1} / \mathrm{S}$ transition. Proc Natl Acad Sci USA 2006; 103: 2695-700. 